\title{
El rol de las universidades peruanas frente a la investigación y el desarrollo tecnológico
}

\section{The Role of Peruvian Universities in the Face of Research and Technological Development}

\author{
Daniela Medina Coronado Universidad César Vallejo, Lima, Perú \\ Universidad Femenina del Sagrado Corazón, Lima, Perú \\ ORCID: https://orcid.org/0000-0002-9180-7613 \\ Magister en Psicología Educativa y Desarrollo Humano en Contextos Interculturales. \\ Docente e Investigadora
}

Recibido 12-05-18 Revisado 25-05-18 Aprobado 26-07-18 En línea 26-09-18

\section{*Correspondencia:}

Email: danmedina27@hotmail.com

\section{Citar como:}

Medina, D. (2018). El rol de las universidades peruanas frente a la investigación y el desarrollo tecnológico- Propósitos y Representaciones, 6(2), 703-737. Doi: http://dx.doi.org/10.20511/ pyr2018.v6n2.244

(C) Universidad San Ignacio de Loyola, Vicerrectorado de Investigación, 2018

(cc) BY-NC-ND Este artículo se distribuye bajo licencia CC BY-NC-ND 4.0 Internacional (http://creativecommons.org/licenses/by-nc-nd/4.0/). 


\section{Resumen}

En el marco de la actual Ley Universitaria 30220 y los procesos que implican su implementación en búsqueda de la calidad de la educación superior universitaria, surge el presente artículo, el que tiene como propósito brindar un acercamiento al lector sobre lo que deberían realizar y lo que vienen haciendo las universidades peruanas para el cumplimiento de una de sus funciones fundamentales, la investigación y el desarrollo tecnológico. Se realiza la revisión de diferentes fuentes documentales a partir de los componentes del quehacer investigativo que refiere el Manual de Frascati: la enseñanza y formación científica, la investigación y desarrollo y los servicios científicos tecnológicos.

Palabas clave: Quehacer investigativo, enseñanza y formación científica, investigación y desarrollo, servicios científicos tecnológicos. 


\section{Summary}

Within the framework of the current University Law 30220 and the processes involved in its implementation in search for quality of higher education, this article is written to introduce the reader to what Peruvian universities are and should be doing to fulfill one of their important roles: research and technological development. Different documentary sources are reviewed based on the components of the research work referred to in the Frascati Manual: scientific teaching and training, research and development and technological scientific services.

Keywords: Research work, scientific teaching and training, research and development, technological scientific services. 


\section{Introducción}

En el siglo XIX Inglaterra fue el principal espacio donde surgieron diversas universidades, tendencia que se extendió a otros países de Europa y a Estados Unidos, bajo el modelo humanista de Wilhelm von Humboldt, la universidad de la edad contemporánea, incorpora la ciencia y la investigación como parte de sus funciones. Del mismo modo pasó en Latinoamérica solo que de manera elitista, siendo la universidad un espacio en que solo las clases dominantes tuvieron acceso a ellas (Báez, 2010).

En la búsqueda de acabar con este elitismo universitario, en el año 1918 surge el movimiento o grito de Córdoba que dio origen a la reforma universitaria en Argentina, movimiento que buscaba democratizar la universidad y darle un carácter científico, teniendo como principal protagonista a la clase media y media baja que anhelaba acceder a las universidades, así como internamente, el reconocimiento del derecho a exteriorizar el pensamiento de los cuerpos universitarios por medio de sus representantes e intervenir en el gobierno, consagrando la autonomía universitaria, promoviendo la participación en el gobierno universitario de los docentes y estudiantes, cuestionando a la universidad como fábrica de títulos profesionales, mostrando preocupación por la innovación pedagógica, buscando la periodicidad y libertad de las cátedras, la gratuidad de la enseñanza y un aspecto relevante la función social de la universidad y compromiso con el cambio que dio como origen a la función de extensión universitaria (Fernández, 2014). En el Perú estas reformas se iniciaron en el año 1900 en la Universidad San Antonio de Abad del Cuzco y en 1919 y 1931 en la Universidad Mayor de San Marcos, teniendo como uno de los grandes ideólogos de la reforma por sus ideales democratizadores de la educación, así como por el cuestionamiento de la formación profesional para el lucro personal sin vínculo con el entorno social a José Antonio Encinas, quien promovió entre estudiantes y docentes el cultivo de ideales que velen por el bien colectivo, por la sociedad, por el país. (Casalino, 2017). 
Estos antecedentes mencionados han sentado las bases de lo que actualmente en las leyes universitarias del mundo se consideran como funciones fundamentales de la universidad: la docencia, la extensión universitaria, la investigación y el desarrollo tecnológico (Fabres, 2005; Sira, 2011). La docencia, como acción de enseñanza aprendizaje de los conocimientos producidos a través de la investigación científica en diversos campos del saber; la extensión universitaria que busca solucionar problemas y satisfacer necesidades del contexto, desde el vínculo existente entre la docencia y la investigación, por medio de acciones concretas. Entonces, la función investigativa es un eje transversal en el quehacer universitario; la universidad es el espacio donde se concretiza la producción, difusión y aplicación de los conocimientos, asimilando los ya generados por la humanidad y produciendo nuevos, a través de las investigaciones de docentes y estudiantes las que tienen como propósito la transformación social. (Organización de las Naciones Unidas para la Educación, la Ciencia y la Cultura, UNESCO, 2008).

Actualmente resulta imposible pensar en dar solución a los problemas más saltantes que presenta la humanidad sin el uso de tecnologías adecuadas a la realidad o sin introducir a la innovación en todos los niveles de la sociedad. Para promover y difundir la importancia del quehacer investigativo, resulta necesario, mejorar la enseñanza de la investigación y generar prácticas institucionales que fomenten el desarrollo de la ciencia y la tecnología para el bien social, involucrándose en ello las diversas estructuras que forman parte de un sistema de innovación; sin estas consideraciones la sociedad puede quedar postrada en el subdesarrollo. Acompañado por este marco general más los procesos de Licenciamiento por el que pasan actualmente las universidades peruanas a partir de la reglamentación de la Ley Universitaria 30220, que surge el presente artículo con el propósito de dar a conocer las actividades que deberían realizar y las que realizan las universidades como parte del cumplimiento de la función investigación y desarrollo tecnológico en el Perú. 


\section{EI quehacer investigativo en la universidad.}

Es preciso iniciar conceptualizando el término quehacer investigativo. Según el manual de Frascati de la OCDE (2002) son actividades científicas y técnicas (ACT) dentro de las universidades, que buscan promover el desarrollo de la ciencia y la innovación para el desarrollo social y constan de tres componentes: enseñanza y formación científica y técnica (STET), investigación y desarrollo (I+D), y servicios científicos tecnológicos (SCT).

La mayoría de universidades en Europa, Asia y América del Norte, desarrollan los 3 componentes de las ACT de forma paralela e inclusive orientadas a labores de extensión, mientras que las universidades latinoamericanas básicamente han centrado sus actividades en la enseñanza y formación científica tanto en pre y posgrado (STET), dejando de lado las otras dos actividades o en su defecto realizándolas de forma tangencial en áreas, institutos o departamentos, con muy pocas implementaciones de las estructuras de interfaz propuestas de forma genérica en los sistemas de ciencia e innovación de sus países, a excepción de países --mencionados en orden de organización y contribución --como Brasil, Argentina, México y Chile.

Entre las principales razones de las marcadas diferencias entre lo que pasa en Europa, Asia y Norteamérica, y las universidades latinoamericanas, se puede considerar que estas últimas rara vez toman en cuenta estándares internacionales en la evaluación de sus procedimientos o resultados, tienen dificultades en el manejo de otros idiomas principalmente el inglés, dan poca atención al impacto de las reformas de otros sistemas, no existen organismos a nivel del estado que regulen de manera seria sus funciones, no se vinculan con otros entornos, no hay voluntad institucional para la inversión, entre otras cuestiones. (UNESCO, 2008).

En el caso peruano, en los últimos años existe una preocupación creciente del gobierno por fortalecer su sistema nacional de innovación, con iniciativas a cargo del Consejo Nacional de Ciencia, Tecnología e 
Innovación Tecnológica (CONCYTEC) y del Ministerio de la Producción, así como de las mismas universidades por dinamizar las actividades ligadas a esta función, producto de acciones del estado para garantizar la calidad de las universidades, con procesos como el Licenciamiento y la Acreditación Universitaria que priorizan la producción académica, la implementación de laboratorios, entre otros aspectos. Tal y como refiere el último Informe Bienal de la Superintendencia Nacional de Educación Superior (2017), a pesar de las críticas que ha tenido por evaluar la calidad de las universidades con solo un indicador: la producción científica, solo 32 universidades de 132 figuran con divulgaciones, destacando la Pontificia Universidad Católica en ingeniería y tecnología, ciencias naturales, ciencias sociales y humanidades, la Universidad Agraria la Molina en ciencias agrícolas y la Universidad Cayetano Heredia en medicina y ciencias de la salud.

A continuación, se brindará información sucinta sobre lo que sucede en el mundo y en el Perú con respecto a cada uno de las tres componentes de las ACT.

\section{Enseñanza y formación científica (STET).}

En universidades nacionales europeas, norte americanas y algunas latinoamericanas, se maneja un sistema en el que los docentes realizan investigación de acuerdo a las asignaturas que enseñan, y acorde a las líneas de investigación de la carrera, hecho que permite tener profesionales altamente especializados, que asesoren y promuevan diversas comunidades académicas, proyectos de investigación con financiamiento interno y externo, pero principalmente realizar clases tan ricas y críticas, no solo a partir de lo que refiere la literatura o la experiencia profesional si no a partir de evidencia empíricas de las propias investigaciones que sirven para rebatir o confirmar lo ya escrito, de tal modo que con respecto a la realidad española, Romaña y Gros (2003, p.9) refieren que "el lugar alcanzado a través de la investigación es lo que permite legitimar las otras prácticas (la gestión y la docencia) ya que es la práctica dominante", sostienen así mismo que los docentes españoles 
cada vez se muestran más proclives a quererse liberar de la docencia para realizar actividades más atractivas como la investigación o la gestión.

Mientras que lo que comúnmente se encuentra en las universidades latinoamericanas es una división entre docentes formadores y docentes investigadores, haciendo ver que son funciones distintas; no obstante, se sostiene el hecho de que no puede existir la docencia sin la investigación. Hoy en día es una tendencia que las universidades soliciten de forma casi obligatoria que los docentes tengan publicaciones producto de sus investigaciones, sin embargo, no se les asigna horas destinadas a esa tarea dentro de su carga laboral o lo que es más grave las universidades no están organizadas en un sistema que les permita hacer investigación. Se supone que docencia e investigación no son actividades separadas, como refiere Bok (1990), lo que se enseña debe cuestionarse, el aula debe funcionar como un laboratorio para el desarrollo de la investigación, con el propósito de ayudar a la solución de diversas necesidades sociales y técnicas, de este modo se genera también un espacio para el trabajo investigativo conjunto entre docentes y estudiantes, conformándose comunidades académicas (Rojas, 2011).

En la vida universitaria se pueden diferenciar dos tipos de vínculo entre la enseñanza y la investigación, una que parte desde el currículo, llamada investigación formativa y otra vinculada a políticas institucionales, gestionada por lo general por los Vicerrectorados de Investigación, llamada formación para la investigación (Parra, 2004; Moreno, 2005; Guerrero, 2007; Miyahira, 2009).

La investigación formativa, definida como el uso de la investigación como herramienta del proceso de enseñanza aprendizaje, busca la difusión de información existente para que el estudiante la incorpore como conocimiento, es dirigida y orientada por un docente, como parte de su función, quien enseña haciendo uso del método de investigación a sujetos en formación, quienes no son investigadores profesionales (Miyahira, 2009). Rojas y Aguirre (2015) sostienen en su estado del arte, que del material revisado se pudo observar 
dos opciones de articulación de la investigación formativa al currículo, "la primera limitada a la enseñanza de la metodología de la investigación y la segunda como componente transversal del currículo" (p.211), es decir, una forma, donde solo hay algunos cursos ligados a la enseñanza de métodos investigativos, y otra en donde a lo largo de la carrera se llevan asignaturas que desarrollan progresivamente habilidades investigativas.

Por lo general, las universidades en Latinoamérica, cumplen más una función profesionalizante, en donde la investigación, como hemos mencionado, se vincula desde el currículo con los procesos de formación profesional, tratando "contenidos relacionados con los métodos y problemas de investigación propios de las disciplinas que sustentan el saber profesional" (Parra, 2004, p. 58), con poca o casi ninguna contribución, en algunos casos, al ejercicio propio de la profesión, es por ello que algunas universidades, según el área del conocimiento, han optado porque sus estudiantes no se titulen con trabajos de investigación que involucren el método científico, sino por ejemplo con planes de negocio, estudios de mercado, entre otras formas de investigar.

Por otro lado, las asignaturas que tienen como propósito dotar de ciertas capacidades investigativas a los estudiantes, en varios países latinoamericanos, incluido el Perú, son impartidas por docentes con poca experiencia en el hacer investigación. Si son docentes a tiempo completo, la distribución de sus horas, están principalmente orientadas a la docencia o a las actividades administrativas, quedando poco espacio para realizar investigación (Parra, 2004). No obstante, actualmente a raíz del proceso de Licenciamiento a cargo de la Superintendencia Nacional de Educación Superior Universitaria, las universidades exigen que sus docentes tengan como mínimo el grado de Magister, así como publicaciones, lo que de algún modo garantiza cierta familiaridad con métodos de investigación, aunque en algunos casos las condiciones para investigar y publicar corren en todo aspecto a cuenta del investigador. 
En cuanto a la formación para la investigación-- la que está sujeta al tipo de profesional que desea formar la universidad-- busca formar profesionales que generen conocimientos, más que solo su uso, siempre bajo el acompañamiento o liderazgo de un docente.

En el Perú, las principales universidades que tienen instalada una estructura y dinámica que les permite formar estudiantes investigadores y que coincidentemente son las que figuran en rankings internacionales de divulgación científica, son la Pontificia Universidad Católica del Perú(PUCP), la Universidad Peruana Cayetano Heredia (UPCH) y la Universidad Mayor de San Marcos (UNMSM) por mencionar las más destacadas. Luego de revisar las web de sus Vicerrectorados de Investigación, se hace referencia de que estas universidades cuentan con diversas actividades para la promoción de los llamados "semilleros de investigación", los que buscan formar a los estudiantes desde el pregrado para el quehacer investigativo, con actividades como: concursos de monografía y tesis con reconocimientos económicos, fondos concursables para el financiamiento de la elaboración de las tesis o de trabajos de investigación entre docentes y estudiantes; así mismo la PUCP cuenta con un programa de apoyo a la iniciación en investigación dirigido a estudiantes quienes desde el tercer año de estudios y bajo la orientación de un docente asesor pueden investigar contando con los recursos necesarios.

Los semilleros de investigación son una estrategia, que tiene una base constructivista y en los métodos activos, que permite a sus participantes, estudiantes y docentes una participación real, priorizando la libertad e innovación para desarrollar aprendizajes, de forma más efectiva que el trabajo formativo en el aula, permitiendo la conformación y desarrollo de grupos y líneas de investigación, así como el vínculo docencia e investigación, fortaleciendo los espacios académicos. Tanto los docentes, como estudiantes, en cualquiera de estas dos situaciones: docentes que decidan asesorar y guiar las investigaciones de sus estudiantes o en su defecto liderar investigaciones con el apoyo de jóvenes investigadores, desarrollarán una serie de competencias que les ayudarán a ser mejores profesionales y a potencializar 
habilidades investigativas; no obstante, cabe aclarar que tal y como hemos visto con las tres universidades mencionadas, esta estrategia requiere de gran apoyo institucional, pues se requiere de recursos económicos, asignación de horas, infraestructura, entre otros aspectos, (Villalba y Gonzáles, 2017).

Otras universidades como la Universidad San Martín de Porres, Universidad San Ignacio de Loyola, Universidad Nacional de Ingeniería, Católica de Santa María, Universidad de Ciencias Aplicadas, por mencionar las que se han hecho más visibles al respecto, con Vicerrectorados de Investigación más recientes, promueven también actualmente los semilleros de investigación en algunas de sus formas, brindando el apoyo institucional respectivo.

\section{Investigación y Desarrollo (I+D).}

Las actividades de innovación y desarrollo, están vinculadas al desarrollo social, la universidad como institución autónoma podría realizar esta labor de forma independiente, no obstante existe un impacto mayor cuando existe la relación universidad - empresa - estado. Sábato y Botana en 1968 fueron quienes concibieron el primer modelo moderno de esta relación, llamado el triángulo de Sábato, en el que se plantea principalmente que las universidades deben interactuar con su entorno, siendo el estado como ente rector, quien plantee las políticas y recursos necesarios para el desarrollo de la capacidad científico tecnológico de un país y las empresas, el motor de empleo y desarrollo de un país, al ofrecer nuevas alternativas de consumo, en productos como servicios a fin de mejorar su productividad y competitividad para permanecer en el mercado. Este primer modelo tuvo acogida en Latinoamérica y en ese marco se crearon diversos centros de desarrollo tecnológico con apoyo del gobierno, luego de esta propuesta surgió en 1998 el modelo de Triple Hélice propuesto por Etzkowitz y Leydesdorff, el que enfatiza en los efectos de las interrelaciones de la triada de Sábato, surgiendo las llamadas spin offs, start ups, spin outs, centros de transferencia de tecnología, entre otras formas de emprendimiento de individuos, grupos 
y organizaciones. A decir de los mismos autores la innovación debe ir unida a la ciencia que produce la universidad y la praxis empresarial, generando el desarrollo económico y tecnológico de las sociedades. (Ramírez \& García, 2010).

Ejemplo de este último modelo, en el Perú, es el spin off "Yacu Tec" de la Universidad de Lima, que ganó en la edición del 2015 de Start Up Perú, el premio de 50,000 soles como inversión para el negocio. Esta iniciativa, se realizó en un trabajo conjunto con el Instituto de Innovación Agraria (INIA) del Ministerio de Agricultura y Riego y consiste en un sistema electroquímico amigable con el medio ambiente, para la producción de ferrato y el uso directo de sus iones en el tratamiento de aguas contaminadas con distintos materiales pesados. Este proyecto, no solo busca el bien social, sino también tiene una perspectiva comercial, como todo spin off, empresas formadas desde la investigación en la universidad (Universidad de Lima, 2016).

Como se observa, el punto de partida es siempre la investigación, los investigadores al realizar su trabajo a partir de la demanda social o del sector productivo, aseguran el desarrollo y progresos económicos, convirtiendo sus trabajos en algo innovador, no solo por su originalidad, sino principalmente porque son funcionales, necesarios y tienen acogida en un sector, generando desarrollo. Lastimosamente en Latinoamérica, los investigadores que pertenecen a la estructura científico tecnológico, suelen vincularse con estructuras fuera de su país de origen, ya que en estos otros espacios, cuentan con los recursos para investigar, son reconocidos y mejor remunerados. Ante esta situación, las universidades interesadas por el tema de investigación y desarrollo han realizado una serie de campañas atractivas para "repatriar" a sus profesionales, por ejemplo en el caso peruano, la Universidad Peruana Cayetano Heredia, tiene el programa llamado "Beca de retorno", el que financia los salarios a jóvenes profesionales que ya poseen grado de Doctor o $\mathrm{PhD}$ por dos años, con el fin de que preparen proyectos de investigación e incrementar su producción científica. Así mismo, como iniciativa del gobierno, cabe mencionar al Programa de Repatriación de Investigadores 
peruanos de Innóvate Perú del Ministerio de Producción, el que permite la incorporación de investigadores a las universidades e institutos públicos de investigación del país.

Retornando a panorama internacional, en los países desarrollados, la gran parte de la investigación y del desarrollo tecnológico se produce en el sector privado y en instituciones de investigación del gobierno, mientras que las universidades que tienen como función principal la investigación, se dedican a atraer y educar investigadores calificados y producir conocimiento académico; por lo general, en estos países se realizan alianzas estratégicas entre el sector privado y las universidades para realizar actividades científico técnicas. India, China, Japón y Corea del Sur son un ejemplo de ello, con un modelo centrado en la investigación en ciencia y tecnología en grandes empresas privadas, no obstante, en los últimos años han invertido en las universidades como centros de investigación, teniendo como referente estándares de las universidades norteamericanas y europeas, en el caso de Japón inclusive superando a las universidades europeas en cuanto a su capacidad de adaptación a las necesidades del mercado (Altbach \& Salmi, 2011).

Siendo conscientes del impacto que tiene la investigación, desarrollo e innovación sobre la economía y la sociedad, los estados europeos vienen generando desde hace varios años políticas e inversiones para la promoción y difusión de la I+D+I (Investigación, desarrollo e innovación) en toda Europa, desarrollada en la actualidad principalmente por organismos públicos, como institutos de investigación, centros tecnológicos y en especial las universidades (Jiménez \& Teba, 2007).

Un espacio donde confluye el trinomio mencionado en párrafos anteriores: universidad-estado-empresaa, son los llamados Parques Tecnológicos, que son una incitativa, por lo general pública que tiene como propósito el crecimiento económico sostenible en el área en el que se instala, para ello se crea un espacio que "promueva y albergue instituciones de investigación, asociadas a universidades del entorno y empresas intensivas 
en conocimiento, entre las que se estimula y produce la transferencia de conocimiento, internamente, pero también con externos" (Gil, 2014, p.5), el concepto se remonta a la década de 1950, con el Stanford Research Park, creado por la Universidad de Stanford en los Estados Unidos, en donde se instalaron General Electric y Hewlett Packard.

Los parques tecnológicos, tuvieron a Silicon Valley como principal referente de lo que significa un sistema virtuoso de innovación, se menciona ello, ya que no es tarea sencilla hacer que este trinomio funcione, por ejemplo en Europa hasta finales del siglo XX hubieron serias dificultades para que la $\mathrm{I}+\mathrm{D}$ pública, se interese en la cooperación empresarial, primando la investigación científica sin orientación al mercado, así es que en el año 1995 en el Informe sobre el libro Verde de la Innovación, la Unión Europea aceptó el fracaso de su sistema de innovación, refiriendo como causa principal la dificultad para convertir el conocimiento en productos y servicios-con excepción de países como Irlanda y Finlandia--a diferencia de Japón y Estados Unidos, sus competidores directos, cuyas políticas y resultados evidenciaban una mejor adaptación al mercado (Romera, 2003).

En Latinoamérica, países que destacan con parques tecnológicos físicos operativos son Brasil con 22 y México con 21 (Gil, 2014). En el caso peruano, existen 10 proyectos, en Arequipa, Piura, La Liberad, Huancayo, Huánuco y Tacna y 4 en Lima, estos últimos como iniciativas de las universidades Cayetano Heredia, Católica del Perú, Ingeniería y San Marcos (Consejo Nacional de Ciencia, Tecnología e Innovación Tecnológica, 2014). Considerando que los Parques Científicos Tecnológicos son una herramienta importante para el vínculo entre la universidad, industria y sociedad a fin de aumentar la competitividad del país y mejorar la calidad de vida, el CONCYTEC apoya el desarrollo de estos parques para que salgan del período de incubación, según información reciente de su web, importante sería revisar qué acciones puntuales, está llevando el estado peruano a fin de promover la implementación de estos parques. 


\section{Conclusiones}

Como producto de la actividad docente e investigadora, las universidades se dotan de diverso equipamiento y personal especializado, en sus laboratorios, Institutos de Investigación y en otras estructuras. Con el fin de maximizar el aprovechamiento social de esos recursos, de ayudar a mantener los equipos, así como de generar ingresos para la universidad, surge la iniciativa de brindar servicios especializados apoyando actividades de $\mathrm{I}+\mathrm{D}+\mathrm{I}$ para clientes internos, así como para diversas entidades públicas como privadas. Al no existir estudios que informen datos cuantitativos o cualitativos al respecto, se revisaron algunas páginas web. Cuentan con este servicio, universidades americanas como Stanford, Harvard, el Instituto Tecnológico de California; en el caso español, la Universidad de Oviedo, Politécnica de Valencia, Politécnica de Madrid, por mencionar algunas. En Latinoamérica, destacan la Universidad de Sao Paulo, Autónoma de México, el Tecnológico de Monterrey y la Universidad de Buenos Aires. Para el caso peruano se revisaron las web de las universidades más destacadas en cuanto a actividad científica y que contaban con información pública en sus páginas web sobre los servicios que prestan. Figuran: la Pontificia Universidad Católica del Perú, que ofrece soluciones a problemas del sector productivo a fin de hacer más competitivo sus productos o servicios, con 40 especialidades, teniendo como caso de éxito a aproximadamente 20 organizaciones; por su parte, la Universidad Nacional de Ingeniería brinda servicios de laboratorio para los sectores: industria, construcción y energía, la Universidad Agraria La Molina brinda capacitación, extensión y servicios en recursos hídricos.

De lo revisado, se infiere que las universidades peruanas aún deben analizar el potencial de sus recursos humanos y de infraestructura, más aun teniendo en cuenta que por los procesos de aseguramiento de la calidad por los que está pasando la universidad peruana, todas, desde aproximadamente el 2016 han implementado sus laboratorios y talleres con la infraestructura necesaria para realizar una formación por competencias, realizando ese análisis, probablemente se pueda ampliar este tipo de servicios a las 
organizaciones, según las especialidades que tenga la universidad, generando recursos adicionales.

\section{Refererencias}

Albatch, P. \& Salmi, J. (2011). The road to academic excellence. The making of word class research universities. Office of the Publisher. The World Bank: Washington DC.

Bok, D. (1990). Universities and the Future of America. London: Duke University Press.

Casalino, C, (2017). Tres etapas y dos personales ilustres en la historia de la Universidad Nacional Mayor de San Marcos. Investigaciones sociales, 21(38), 173-186.

Fabre, C. (febrero del 2005). Las funciones sustantivas de la universidad y su articulación en un departamento docente. Resumen presentado en el V Congreso Internacional Virtual de Educación. Universidad Agraria de La Habana Cuba.

Fernández, N. (2014). Universidad, sociedad y conocimiento reflexiones para el debate. Avaliação, 19(3), 663-687. Recuperado de: http://submission. scielo.br/index.php/aval/article/view/141224

Gil, R. (2014). Los Parques Científico Tecnológicos en América Latina: Análisis de la situación actual. Recuperado de: http://vcentrum.pucp. edu.pe/investigacion/wps/pdf/CEFE_WP2014-05-0008.pdf

Guerrero. M. E. (2007). Formación de habilidades para la investigación desde el pregrado. Acta colombiana de Psicología, 10(2), 190-192.

Jiménez, G., \& Teba J. (2007). Parques científico tecnológicos y su importancia en los sistemas regionales de innovación. Recuperado de: https://eco.mdp.edu.ar/cendocu/repositorio/01008.pdf

Miyahira, J.M. (2009). La investigación formativa y la formación para la investigación en el pregrado. Revista Médica Herediana, 20(3), 119-122.

Moreno, M. G. (2005). Potenciar la educación. Un currículum transversal de formación para a investigación. Revista electrónica Iberoamericana 
sobre calidad, eficacia y cambio en educación, 3(1). Recuperado de: https://repositorio.uam.es/handle/10486/660897

Organización para la Cooperación y Desarrollo Económicos, OCDE. (2002). Medición de las actividades cientificas y tecnológicas. Propuesta de norma práctica para encuestas de investigación y desarrollo experimental. Manual de Frascati. Fundación Española Ciencia y Tecnología: París.

Organización de las Naciones Unidas para la Educación, la Ciencia y la Cultura, UNESCO. (2008). Universidad y desarrollo en Latinoamérica: experiencias exitosas de centros de investigación. Panamericana formas e impresos: Bogotá.

Parra, C. (2004). Apuntes sobre la investigación formativa. Educación y educadores, 7, 57- 77. Recuperado de: http://educacionyeducadores. unisabana.edu.co/index.php/eye/article/view/549

Ramírez, M., \& García, M. (2010). La alianza Universidad - Empresa Estado: una estrategia para promover la innovación. Revista EAN, 68, 112. Doi: https://doi.org/10.21158/01208160.n68.2010.500

Rojas, C., \& Aguirre, S. (2015). La formación investigativa en la educación superior en América Latina y el Caribe: una aproximación a su estado del arte. Revista Eleuthera, 12, 197-222. https://doi.org/10.17151/ eleu.2015.12.11

Rojas, H.M. (2011). Docencia y formación científica universitaria. Magis. Revista internacional de investigación en educación, 4(7), 121-136.

Romaña, T., \& Gros, B. (2003). La profesión del docente universitario del siglo XXI: ¿Cambios superficiales o profundos? Revista de enseñanza universitaria, 21, 7-35. Recuperado de: http://hdl.handle. net/11441/54876

Romera, F. (2003). Los parques científico tecnológicos, sistemas virtuosos de innovación. Economía industrial.345, 85-102.

Sira, S. (2011). Equilibrio entre las funciones de docencia, investigación y extensión en Ingeniería. Desarrollo Histórico. Ingeniería y Sociedad, 6(11), 51-57. Recuperado de: http://servicio.bc.uc.edu.ve/ingenieria/ revista/IngenieriaySociedad/a6n1/art5.pdf 
Superintendencia Nacional de Educación Superior (2017). Informe bienal sobre la realidad universitaria peruana. Recuperado de: http://repositorio.minedu.gob.pe/bitstream/handle/MINEDU/5716/ Informe $\% 20$ bienal $\% 20$ sobre $\% 201 \mathrm{a} \% 20$ realidad $\% 20$ universitaria $\% 20$ peruana.pdf? sequence $=1 \&$ isAllowed $=\mathrm{y}$

Universidad de Lima (23 de marzo del 2016). Primer spin off de la Ulima triunfa en Start Up Perú. Instituto de Investigación Cientifica. Recuperado de: http://www.ulima.edu.pe/instituto-de-investigacioncientifica/noticias/primer-spin-de-la-ulima-triunfa-en-start-peru

Villalba, J.C., \& Gonzáles A. (2017). La importancia de los semilleros de investigación. Revista Prolegómenos -Derechos y Valores, 20 (39), 9-10,133. Doi: https://doi.org/10.18359/prole.2719 